\title{
Fever Unmasking the Brugada Syndrome in a Patient with COVID-19: A Case Report
}

\author{
Mohamed Mohamedali ${ }^{1}$, Mahmood Al-Jabry ${ }^{1}$, Riswan Abdulmajeed ${ }^{1}$, Fahmi Othman ${ }^{1}$, \\ Mohammed Danjuma ${ }^{1}$, Mohamed Yassin ${ }^{1}$, mohamed aboukamar ${ }^{1}$, Ahmad Abujaber ${ }^{1}$, and \\ Abdulqadir Nashwan ${ }^{1}$ \\ ${ }^{1}$ Hamad Medical Corporation
}

July 28, 2020

\begin{abstract}
The effect of COVID-19 is not limited to the respiratory system. Patients who are at risk to develop cardiac arrhythmias might be affected especially when unmasked with fever. A 41-year-old Bangladeshi male patient presented with frequent palpitations and dizziness. His ECG revealed Brugada pattern while being diagnosed with COVID-19.
\end{abstract}

Fever Unmasking the Brugada Syndrome in a Patient with COVID-19: A Case Report

Mohamed G. Mohamedali ${ }^{1}$, Mahmood M. Al-Jabry ${ }^{2}$, Riswan P. Abdulmajeed ${ }^{3}$, Fahmi S. Othman ${ }^{4}$, Mohammed I. Danjuma ${ }^{5}$, Mohamed A. Yassin ${ }^{6}$, Mohamad R. Abou Kamar ${ }^{7}$, Ahmad A. Abujaber ${ }^{8,10}$, Abdulqadir J. Naswhan ${ }^{9,10}$

1. Consultant, Hazm Mebaireek General Hospital (HMGH), Hamad Medical Corporation (HMC), Doha, Qatar.

2. Director of Nursing for Emergency Department, Hazm Mebaireek General Hospital (HMGH), Hamad Medical Corporation (HMC), Doha, Qatar.

3. Charge Nurse, Emergency Department, Hazm Mebaireek General Hospital (HMGH), Hamad Medical Corporation (HMC), Doha, Qatar.

4. Cardiology Consultant, Hazm Mebaireek General Hospital (HMGH), Hamad Medical Corporation (HMC), Doha, Qatar.

5. Consultant, Hamad General Hospital (HGH), Hamad Medical Corporation (HMC), Doha, Qatar.

6. Consultant, Department of Medical Oncology, National Center for Cancer Care \& Research, Hamad Medical Corporation (HMC), Doha, Qatar.

7. Consultant, Department of Infectious Diseases, Communicable Disease Center (CDC), Hamad Medical Corporation (HMC), Doha, Qatar.

8. Assistant Executive Director of Nursing, Hazm Mebaireek General Hospital (HMGH), Hamad Medical Corporation (HMC), Doha, Qatar.

9. Director of Nursing for Education \& Practice Development, Hazm Mebaireek General Hospital (HMGH), Hamad Medical Corporation (HMC), Doha, Qatar.

10. Adjunct Senior Instructor, University of Calgary in Qatar (UCQ), Doha, Qatar.

Corresponding author: Abdulqadir J. Nashwan

Nursing861@gmail.com

Tel: (+974) 40240487 
Mob: (+974) 66473549

P.O.Box 3050 Doha, Qatar

www.hamad.qa

\section{Hosted file}

CaseReport_BS_v0.2.docx available at https://authorea.com/users/346661/articles/472590-feverunmasking-the-brugada-syndrome-in-a-patient-with-covid-19-a-case-report

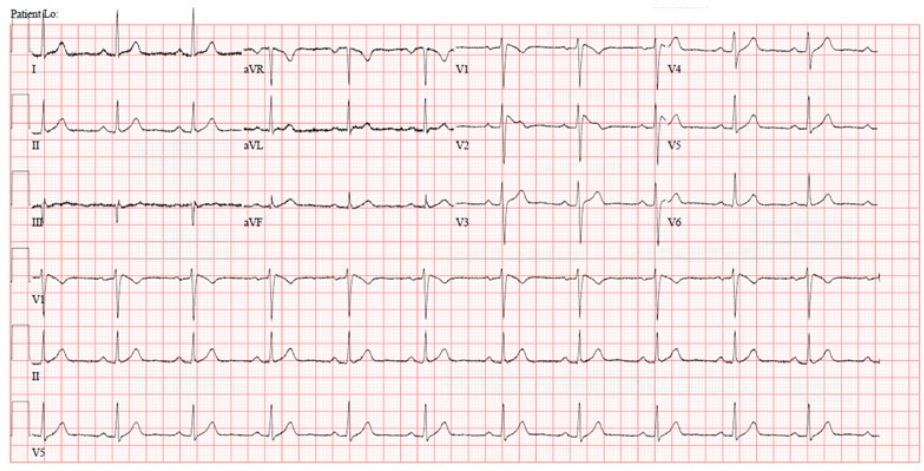

Figure 1. Initial 12-lead ECG upon admission to ED. 


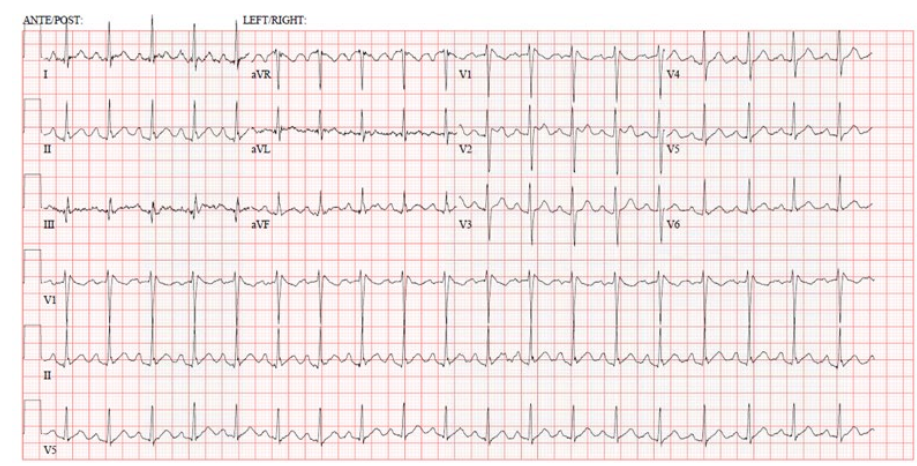

Figure 2. Repeated 12-lead ECG after 2 days. 